\title{
Detection of Focal Longitudinal Changes in the Brain by Subtraction of MR Images
}

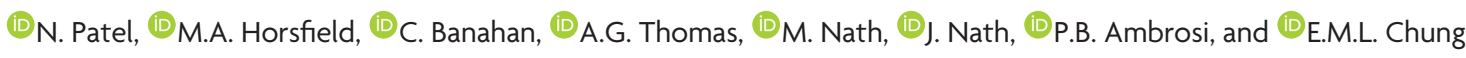

\begin{abstract}
BACKGROUND AND PURPOSE: The detection of new subtle brain pathology on MR imaging is a time-consuming and error-prone task for the radiologist. This article introduces and evaluates an image-registration and subtraction method for highlighting small changes in the brain with a view to minimizing the risk of missed pathology and reducing fatigue.
\end{abstract}

MATERIALS AND METHODS: We present a fully automated algorithm for highlighting subtle changes between multiple serially acquired brain MR images with a novel approach to registration and MR imaging bias field correction. The method was evaluated for the detection of new lesions in 77 patients undergoing cardiac surgery, by using pairs of fluid-attenuated inversion recovery MR images acquired 1-2 weeks before the operation and 6-8 weeks postoperatively. Three radiologists reviewed the images.

RESULTS: On the basis of qualitative comparison of pre- and postsurgery FLAIR images, radiologists identified 37 new ischemic lesions in 22 patients. When these images were accompanied by a subtraction image, $46 \mathrm{new}$ ischemic lesions were identified in 26 patients. After we accounted for interpatient and interradiologist variability using a multilevel statistical model, the likelihood of detecting a lesion was 2.59 $(95 \% \mathrm{Cl}, 1.18-5.67)$ times greater when aided by the subtraction algorithm $(P=.017)$. Radiologists also reviewed the images significantly faster $(P<.001)$ by using the subtraction image (mean, 42 seconds; $95 \% \mathrm{Cl}, 29-60$ seconds) than through qualitative assessment alone (mean, 66 seconds; $95 \% \mathrm{Cl}$, 46-96 seconds).

CONCLUSIONS: Use of this new subtraction algorithm would result in considerable savings in the time required to review images and in improved sensitivity to subtle focal pathology.

$I^{\mathrm{n}}$ ncreasingly, diagnostic radiologic assessment does not involve a single examination at 1 time point but makes use of follow-up imaging for monitoring responses during the course of treatment or to assess the progression of a condition with a view to intervention. ${ }^{1-3}$ Manual interpretation of this type of data is burdensome and prone to errors. A challenge for medical image analysts is the development of reliable techniques to assist the radiologist. ${ }^{4}$ Eas-

Received May 11, 2015; accepted after revision December 14, 2016.

From the Department of Cardiovascular Sciences (N.P., M.A.H., M.N., J.N., E.M.L.C.), University of Leicester, Leicester Royal Infirmary, Leicester, UK; Leicester National Institute of Health Research Cardiovascular Biomedical Research Unit (N.P.,

E.M.L.C.), Glenfield Hospital, Leicester, UK; Departments of Radiology (A.G.T.,

P.B.A.) and Medical Physics (C.B., E.M.L.C.), University Hospitals of Leicester Na-

tional Health Service Trust, Leicester, UK; and Neuri Beaujon (P.B.A.), University Paris Diderot, Paris, France.

This study was funded by the British Heart Foundation (FS/10/46/288350). N. Patel was funded by the National Institute of Health Research Leicester Cardiovascular Biomedical Research Unit.

Please address correspondence to Emma M.L. Chung, MD, Department of Cardiovascular Sciences, University of Leicester, Leicester Royal Infirmary, LE2 7LX, UK; e-mail: emlc1@leicester.ac.uk

-- Indicates open access to non-subscribers at www.ajnr.org

http://dx.doi.org/10.3174/ajnr.A5165 ier and faster detection of pathologic changes facilitates diagnosis and makes it more cost-effective to monitor disease evolution in the long term.

Manual analysis of medical images acquired at different time points is generally limited to a qualitative comparison. The repositioning of the patient is never identical, the scanning equipment used may be different or may have been upgraded, the acquisition parameters may have altered between scans, and local shape deformations of nonrigid anatomic structures occur. The expert radiologist compensates intuitively for some of these unwanted differences with knowledge of anatomy and the expected signal differences to identify and reject certain artifactual changes.

One of the major artifacts associated with MR imaging is caused by the spatially nonuniform transmission and reception properties of the radiofrequency coils. ${ }^{5}$ Commonly, a large transmitter coil built into the bore of the magnet is used for radiofrequency transmission, resulting in relatively uniform transmission properties. However, to improve the signal-to-noise ratio, an array of small coils is used for signal reception, and these have very spatially nonuniform reception properties. ${ }^{6}$ It is common to correct for this by mapping the reception properties of the coils and 


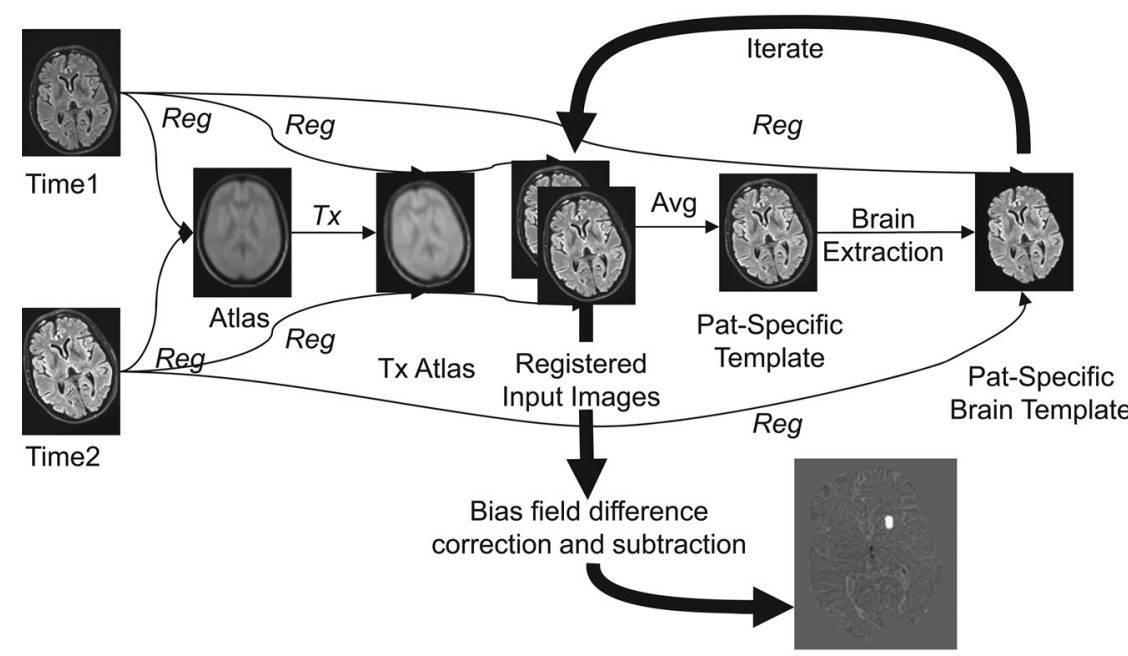

FIG 1. Flow chart illustrating the processing steps to produce a subtraction image for 2 images acquired at different time points. Registration $(R e g)$ is first done with a generic atlas target to determine the average position of the input images. This atlas is transformed to the average position of the input images and serves as the registration target for the second registration. The registered input images are averaged to create a patient-specific target at the average position. This target is then refined by brain extraction and further registration of the input images to it. After the final registration, the differences in the bias field are corrected; then, a subtraction highlights any focal changes in the input images between time points. Tx indicates a transform/ transformation.

rescaling the image intensities pixel by pixel. ${ }^{7}$ This mapping, however, is based on a number of assumptions and does not remove the image-intensity shading, known as the bias field, completely. It is also possible to perform a bias field correction as a postprocessing step, but again this is based on a number of assumptions about the nature of the field and its stability. ${ }^{8,9}$ The algorithm described in the current work circumvents these issues by correcting the bias field in subtraction images rather than in the source images themselves.

This article describes an image registration and subtraction technique that can highlight local changes in MR images of the brain acquired at $\geq 2$ time points. It is robust to changes in the patient position and in the bias field. The technique was evaluated by using a set of 77 patients who were scanned before and after cardiac surgery, with the aim of assessing whether this algorithm could aid in the detection of small ischemic brain lesions resulting from emboli shed from the vascular system as a result of an operation. ${ }^{10}$ The value of the system was assessed by examining the lesion detection rate and time required for the assessment of scans for radiologists examining pairs of images with and without the aid of our subtraction method.

\section{MATERIALS AND METHODS \\ Registration and Subtraction}

The method is based on registration of images acquired at different points in time, the correction of any differences due solely to radiofrequency reception nonuniformity, and subsequent subtraction to reveal any differences due to focal pathology. The method is described in detail elsewhere ${ }^{11}$ and is illustrated in Fig 1. Briefly, input images are registered to a common frame of reference at the average position of all input images. Then, radiofrequency bias field variations are modeled as a smoothly varying polynomial function; then, individual registered images are cor- rected by pixel-wise division by the modeled field. Finally, individual pairs of registered and corrected images are subtracted (Fig 2).

\section{Patients}

We examined data from patients enrolled in a clinical study of neurologic deficits following cardiac surgery, as detailed elsewhere. ${ }^{10}$ All clinical data were collected in accordance with local research ethics procedures. All patients provided written informed consent for their anonymized data to be used in this research.

\section{MR Imaging}

MR imaging was performed by using a 3T system (Magnetom Skyra; Siemens, Erlangen, Germany) with a 20-channel phased array head and neck coil for signal reception. MR imaging studies were performed 1-2 weeks before cardiac surgery and repeated 6-8 weeks after surgery to identify any new postoperative ischemic lesions. The same scanning protocol was used on both occasions, except that the angiogram was not obtained postsurgery. After a localizer image, the scanning protocol consisted of a diffusion-weighted image, time-of-flight angiography, a susceptibility-weighted image, and a FLAIR image. ${ }^{12}$ The total imaging time was approximately 30 minutes.

For this study, only the FLAIR images were analyzed. The FLAIR images consisted of 48, 3-mm-thick contiguous axial sections positioned parallel to the anterior/posterior commissure line. The $k$-space matrix size was $320 \times 224$, zero-filled to give an image matrix of $320 \times 320$ and an FOV of $240 \times 240 \mathrm{~mm}(\mathrm{TR} /$ TE/TI, 6770/108/2170 ms).

\section{Image Review and Analysis}

Images were analyzed independently by 3 experienced neuroradiologists in 2 stages. First, for each patient, the pre- and postsurgery FLAIR images were presented side by side on a computer display and the radiologist paged through both the images section by section to identify any new ischemic lesions. The anatomic regions of any new lesions were also noted. Signal hyperintensity clearly extending outside the brain parenchyma or minor variations in ependymal signal hyperintensity (commonly encountered on FLAIR sequences) were considered artifactual. A digital timer was used by each neuroradiologist to note the time taken to review images from each patient.

Second, for each patient, the coregistered pre- and postsurgery FLAIR images along with the difference image were presented. The neuroradiologist paged through all 3 images simultaneously section by section to identify any new ischemic lesions on the difference image, with the pre- and postsurgery images used to confirm or refute the findings. Thus, it is unlikely that there were any false-positive findings. A subsequent review of the original 


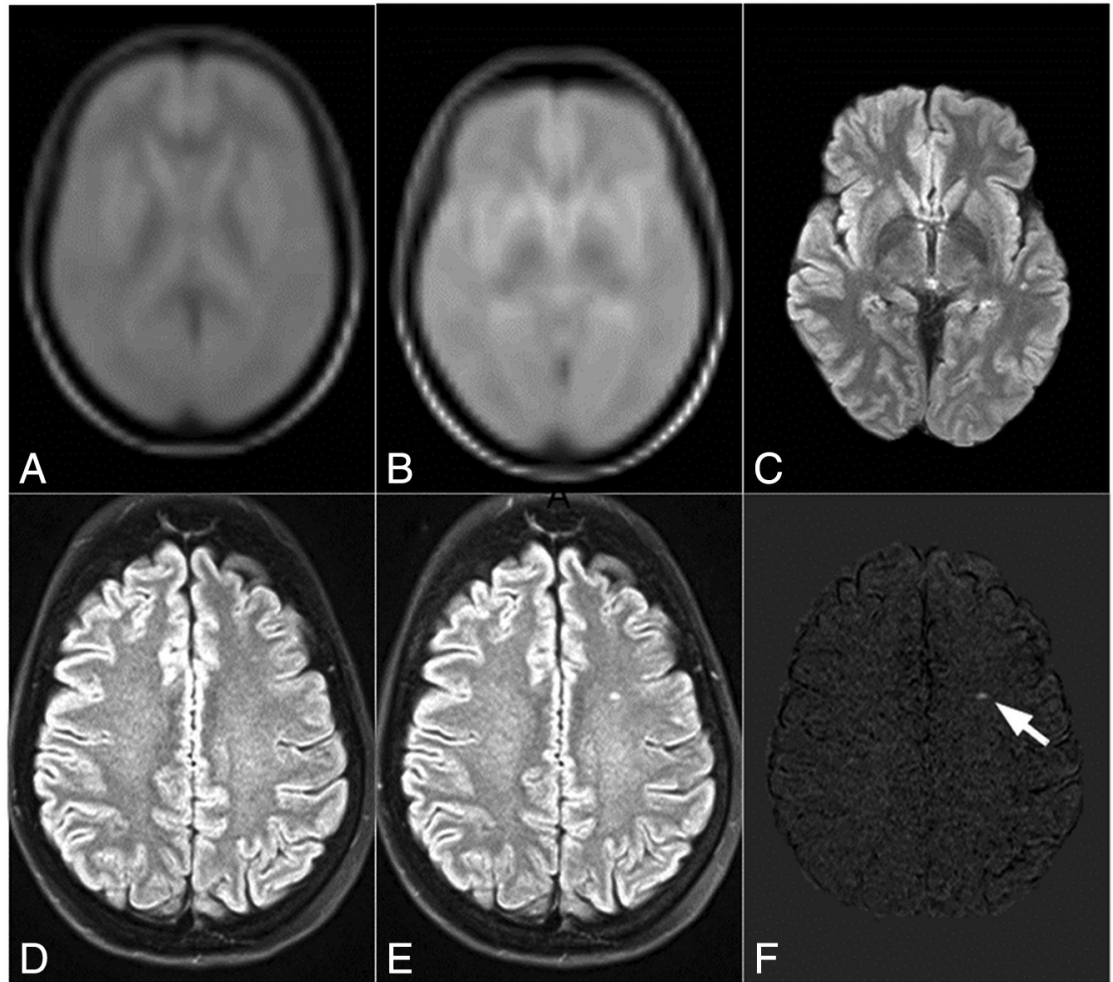

FIG 2. Images to illustrate the processing steps for registration and subtraction. A, Generic proton-density-weighted registration target. $B$, The generic template transformed to the average position of the input images. $C$, The final patient-specific registration target formed by intensityaveraging the input images registered to the template at the target position and after skullstripping. More superior sections of the first $(D)$ and second $(E)$ input images, registered to the patient-specific registration target. $F$, The difference between the second-input image and the first-input image after correction for differences in their bias fields. The arrow indicates a new lesion, which was present at follow-up but absent on the baseline image.

FLAIR images confirmed that lesions originally overlooked were visible in retrospect.

The time required to perform each type of analysis was recorded. There was a gap of at least 4 weeks between the first and second analysis for any given patient, and patient scans were presented in an arbitrary order to avoid familiarity with the images.

\section{Statistical Analysis}

The total evaluation time and the number of lesions detected in 77 patients by 3 radiologists were compared for both the qualitative and subtraction-aided review methods. We assumed that the total number of lesions follows a Poisson distribution and modeled our results with a generalized linear mixed model. The model included the method of review (2 levels: qualitative- and subtraction-aided review) as a fixed effect, individual patient and radiologist as random effects, and the natural logarithm function as a link function. To assess sensitivity to detect the presence of a lesion, we created a binary variable to represent the presence or absence of a lesion with a value of 1 when the total number of lesions was greater than zero, and zero otherwise. We assumed that this binary variable follows a Bernoulli distribution and modeled the presence of lesion data by using a generalized linear mixed model approach (logistic generalized linear mixed model). The logistic generalized linear mixed model included the logit function as a link function and the same fixed and random effects structure as described for the Poisson generalized linear mixed model.

To account for increased variability with the mean review time, we transformed the data on total processing time by using the natural logarithm function, and the transformed data were analyzed by using a linear mixed model incorporating the method of review as a fixed effect and individual patient and radiologist as random effects. For all models, a predictor was considered statistically significant if the 2-sided type I error rate was $<5 \%$ (ie, $P<.05$ ). All statistical analyses were performed by using R statistical and computing software, Version 3.3 (http://www.r-project.org/) with appropriate packages (lme4, ggplot2).

\section{RESULTS}

Pairs of images from 77 patients (72 men; mean age, $62.8 \pm 10.4$ years; range, 32-80 years) were evaluated independently by 3 radiologists. These 77 patients had postoperative MR imaging performed at a mean of $7 \pm 1.25$ weeks after surgery. Five of the 77 (7\%) patients had perioperative strokes: Patients 12 and 61 had a lacunar infarct in the right corona radiata, patient 46 had a lacunar infarct in the left corona radiata, patient 18 had a lacunar infarct in the right frontal lobe, and patient 62 had 2 small lacunar infarcts in the right superior parietal lobule and the left medial percentual gyrus.

With just the pre- and postsurgery FLAIR images, on average, 37 new ischemic lesions were identified in 22 patients (median, 1 lesion per patient; range, 1-5 lesions). The anatomic locations of these new lesions were the following: left frontal WM (18 lesions); right frontal WM (9 lesions); right parieto-occipital subcortical WM (2 lesions); left corona radiata (2 lesions); left centrum semiovale (1 lesion); left anterior striatum (2 lesions); and left (1 lesion) and right (2 lesions) cerebellum. Lesions missed during qualitative assessment were frequently subsequently identified among preexisting white matter hyperintensities.

Using the difference image in combination with the pre- and postsurgery FLAIR images, we identified an average of 46 new ischemic lesions in 26 patients (median, 1 lesion per patient; range, 1-8 lesions). Therefore, the number of new ischemic lesions identified increased by $24 \%$, and the number of patients who had new lesions increased by $18 \%$ when review was aided by a subtraction image. The anatomic locations of these new lesions were the following: left frontal WM (20 lesions); right frontal WM (9 lesions); left (1 lesion) and right (1 lesion) parieto-occipital subcortical WM; right parietal WM (1 lesion); left temporal lobe (2 lesions); left (2 lesions) and right (1 lesion) corona radiata; left centrum semiovale (2 lesions); left parietal cortex (1 lesion); left 


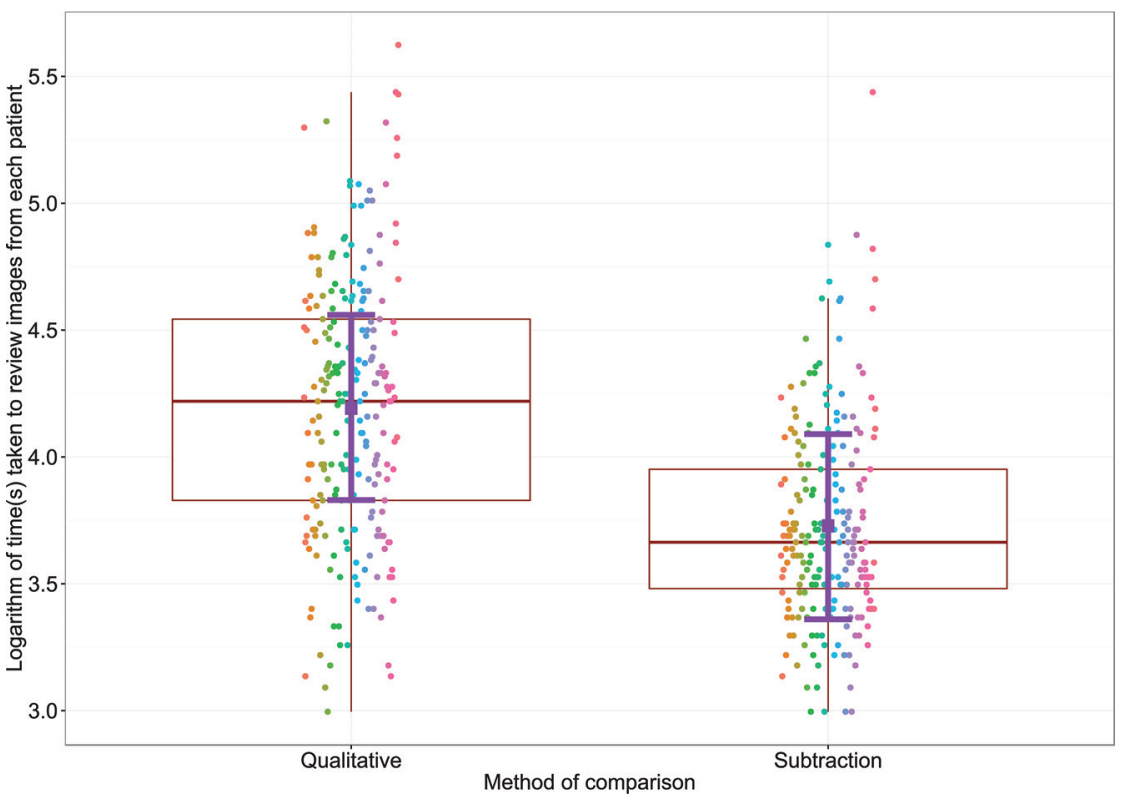

FIG 3. The logarithm of time (seconds) taken to process the data by qualitative comparison and aided by the image-subtraction algorithm. The scatterplots show the triplicate log-transformed time data (of 3 radiologists) for each patient along with boxplots presenting the median, lower, and upper quartiles for each algorithm. Points with the same color represent data from the same patient. The plot also presents the predicted mean time (square marker) along with corresponding $95 \%$ confidence intervals (error bar) for both methods obtained from a linear mixed model fit to the data.

anterior striatum (2 lesions); pons (1 lesion); superior vermis (1 lesion); and right (2 lesions) cerebellum.

Most lesions identified by qualitative assessment were also identified when aided by the subtraction algorithm. The exceptions were 1 lesion in the left and 2 in the right frontal WM and 1 lesion in the left and 1 in the right cerebellum. On further examination of the original FLAIR images, the lesions in the frontal white matter were very small $(<1 \mathrm{~mm})$ and of a level similar to that of the background "mottle." These were confirmed by only 1 of the 3 radiologists. The cerebellar lesions not appreciated on the subtraction image were larger and were definitely present on repeat viewing.

When we pooled the data over all radiologists, the mean lesion counts were 67 and 80 for the qualitative and subtractive methods, respectively, though there was no evidence that the mean lesion counts were significantly different between methods $(P=$ .101). When we accounted for the variabilities between patients and radiologists, the logistic generalized regression model suggested that the likelihood of detecting the presence of a lesion was 2.59 (95\% CI, 1.18-5.67) times greater with the subtraction compared with the qualitative method $(P=.017)$.

When we accounted for the variability in the data between patients (interpatient variability) and between radiologists (interradiologist variability), the mean time to review the images was significantly $(P<.001)$ lower with the subtraction algorithm (mean, 42 seconds; 95\% CI, 29-60 seconds) compared with qualitative assessment (mean, 66 seconds; 95\% CI, 46-96 seconds), reducing the processing time by $37 \%$. Estimates of between-patient variability were slightly higher $(0.27 ; 95 \% \mathrm{CI}, 0.22-0.34)$ than between-radiologist variability $(0.17 ; 95 \% \mathrm{CI}, 0.07-0.42)$. Figure 3 presents the log-transformed time data (of 3 radiologists) for each patient alongside the predicted mean time for both algorithms obtained from the linear mixed-model fit to the data.

\section{DISCUSSION}

We have demonstrated a method for the registration, subtraction, and bias field correction of serial MR images, which can be applied in longitudinal patient studies. The subtraction image highlights the presence of new lesions, which can be confirmed or refuted on closer inspection of the source images. The method is tailored toward robust detection of focal differences in pathology that develop or resolve between scans.

Axial FLAIR imaging frequently has CSF flow-related artifacts in the posterior fossa, which necessarily result in a more heterogeneous parenchymal background on the subtraction image. On the subtraction image, the preexisting lesions were removed, but new lesions were highlighted as hyperintense, thus aiding detection. Similarly, posterior fossa lesions on FLAIR are sometimes difficult to separate from adjacent flowrelated signal hyperintensity and can be easily overlooked, particularly at the periphery of the cerebellum.

Although several other studies have previously used subtraction imaging, ${ }^{13-18}$ the procedure outlined here has several advantages. First, it can be used to analyze MR imaging data acquired serially over many time points in an unbiased fashion. Most previous studies have either selected one of the image time points as the registration target ${ }^{16}$ or have been restricted to showing the differences between pairs of images by realigning them to a position half-way between the 2 scans. ${ }^{18}$ By transforming all of the images to their average position, the current method allows the alignment of any number of image time points. This is achieved by first registering them to a generic atlas target to facilitate relative positioning.

Second, previous studies have incorporated bias field correction by using, for example, the N3 method, ${ }^{9}$ in which the input images are individually bias-corrected as the first processing step. However, the N3 method is prone to errors in the bias field correction scheme, leading to residual shading artifacts in the subtracted images. The current method provides an alternative correction scheme that relies on only the assumption that in the absence of bias field differences, tissues that are not involved in focal pathologic changes should remain substantially unchanged in intensity between image acquisitions. Thus, the differences in the bias field between scans can be estimated and used to correct the subtracted images.

We believe that this method could provide a useful tool for radiologists when reviewing serially acquired MR images. First, the time required to review the images was reduced by approximately $37 \%$ when using the registered and subtracted images. All 
processing was fully automatic and therefore could be performed automatically as an adjunct to the acquisition of new scans and retrieval of previous scans from a PACS system.

Second, the number of new ischemic lesions identified increased by $24 \%$, and the number of patients who had new lesions increased by $18 \%$ when using the subtraction images compared with simply viewing the 2 scans. The likelihood of observing a lesion was also 2.6 times greater when using the new algorithm. These represent substantial increases in sensitivity in detecting new ischemic tissue damage. Although this pathology consisted of relatively small ischemic lesions and only new pathology was identified, subtle changes in tissue in other diseases, such as MS, might be equally identified and could prove important in monitoring disease progression or stability and guiding treatment decisions. ${ }^{19}$

On the other hand, when using the subtraction images, 2 lesions in the cerebellum were missed. On subsequent review, these lesions were clearly present in the view of the radiologists.

The 2 scans for each patient were acquired approximately 8-10 weeks apart, so there was little chance for more substantial tissue changes to develop or for cerebral atrophy to advance. We used a rigid-body (translation and rotation) registration to align the 2 scans, which proved adequate in all cases. More sophisticated nonlinear registration ${ }^{20}$ would be required for longer follow-up periods or to account for more profound structural changes in the brain, for example, in the follow-up of patients with brain tumor and after an operation or in cases of severe atrophy.

To provide an unbiased image registration, the method uses a generic atlas in the first processing step. Because the atlas is used only to find the approximate head position for the serially acquired images, it is likely that other generic atlases ${ }^{21,22}$ could also be used without compromising the quality of the final registration. The scan parameters were kept constant across both scans because the method is unlikely to work well when there are gross differences in contrast between the 2 images. Constraining the scan parameters was easy to do in the context of our study but might be more problematic when patients are rescanned in a routine setting, possibly with different scanner types from different manufacturers. On the other hand, particularly if a $3 \mathrm{D}$ acquisition is used, the method should be relatively robust to gross changes in the patient's head position because of the novel bias field correction used. However, the effects of head position and scanner settings would need to be assessed in future studies.

\section{CONCLUSIONS}

We have developed a fully automated image registration and subtraction scheme capable of highlighting small changes in serial MR imaging scans of the brain. The method should now be used in larger studies to investigate its clinical potential in monitoring progression in different diseases and for longer time periods.

Disclosures: Mark A. Horsfield-UNRELATED: Stock/Stock Options: Xinapse Systems Ltd, Comments: director and stock holder. Emma Chung—RELATED: Grant: British Heart Foundation, Comments: held an Intermediate Basic Science Research Fellowship funded by the British Heart Foundation. * Money paid to the institution.

\section{REFERENCES}

1. Longstreth WT Jr, Arnold AM, Kuller LH, et al. Progression of magnetic resonance imaging-defined brain vascular disease predicts vascular events in elderly: the Cardiovascular Health Study. Stroke 2011;42:2970-72 CrossRef Medline

2. Rovira A, Auger C, Alonso J. Magnetic resonance monitoring of lesion evolution in multiple sclerosis. Ther Adv Neurol Disord 2013; 6:298-310 CrossRef Medline

3. Alpert NM, Berdichevsky D, Levin Z, et al. Performance evaluation of an automated system for registration and postprocessing of CT scans. J Comput Assist Tomogr 2001;25:747-52 CrossRef Medline

4. Castellino RA. Computer aided detection (CAD): an overview. 2005; 23;5:17-19 Medline

5. Vovk U, Pernus F, Likar B. A review of methods for correction of intensity inhomogeneity in MRI. IEEE Trans Med Imaging 2007;26: 405-21 CrossRef Medline

6. Roemer PB, Edelstein WA, Hayes CE, et al. The NMR phased array. Magn Reson Med 1990;16:192-225 CrossRef Medline

7. Vemuri P, Kholmovski EG, Parker DL, et al. Coil sensitivity estimation for optimal SNR reconstruction and intensity inhomogeneity correction in phased array MR imaging. Inf Process Med Imaging 2005;19:603-14 Medline

8. Wicks DA, Barker GJ, Tofts PS. Correction of intensity nonuniformity in MR images of any orientation. Magn Reson Imaging 1993;11: 183-96 CrossRef Medline

9. Sled JG, Zijdenbos AP, Evans AC. A nonparametric method for automatic correction of intensity nonuniformity in MRI data. IEEE Trans Med Imaging 1998;17:87-97 CrossRef Medline

10. Patel N, Horsfield MA, Banahan C, et al. Impact of perioperative infarcts after cardiac surgery. Stroke 2015;46:680-86 CrossRef Medline

11. Horsfield MA, Rocca MA, Pagani E, et al. Estimating brain lesion volume change in multiple sclerosis by subtraction of magnetic resonance images. J Neuroimaging 2016;26:395-402 CrossRef Medline

12. Taoka T, Iwasaki S, Nakagawa $H$, et al. Fast fluid-attenuated inversion recovery (FAST-FLAIR) of ischemic lesions in the brain: comparison with T2-weighted turbo SE. Radiat Med 1996;14:127-31 Medline

13. Bosc M, Heitz F, Armspach JP, et al. Automatic change detection in multimodal serial MRI: application to multiple sclerosis lesion evolution. Neuroimage 2003;20:643-56 CrossRef Medline

14. Meier DS, Guttmann CR. Time-series analysis of MRI intensity patterns in multiple sclerosis. Neuroimage 2003;20:1193-209 CrossRef Medline

15. Tan IL, van Schijndel RA, Fazekas F, et al. Image registration and subtraction to detect active $\mathrm{T}(2)$ lesions in MS: an interobserver study. J Neurol 2002;249:767-73 CrossRef Medline

16. Duan Y, Hildenbrand PG, Sampat MP, et al. Segmentation of subtraction images for the measurement of lesion change in multiple sclerosis. AJNR Am J Neuroradiol 2008;29:340 - 46 CrossRef Medline

17. Ganiler O, Oliver A, Diez Y, et al. A subtraction pipeline for automatic detection of new appearing multiple sclerosis lesions in longitudinal studies. Neuroradiology 2014;56:363-74 CrossRef Medline

18. Battaglini M, Rossi F, Grove RA, et al. Automated identification of brain new lesions in multiple sclerosis using subtraction images. $J$ Magn Reson Imaging 2014;39:1543-49 CrossRef Medline

19. Fazekas F, Soelberg-Sorensen P, Comi G, et al. MRI to monitor treatment efficacy in multiple sclerosis. J Neuroimaging 2007;17(suppl 1):50S-55S CrossRef Medline

20. Sotiras A, Davatzikos C, Paragios N. Deformable medical image registration: a survey. IEEE Trans Med Imaging 2013;32:1153-90 CrossRef Medline

21. Mazziotta J, Toga A, Evans A, et al. A probabilistic atlas and reference system for the human brain: International Consortium for Brain Mapping (ICBM). Philos Trans R Soc Lond B Biol Sci 2001;356: 1293-322 CrossRef Medline

22. Xing W, Nan C, Zuo Z, et al. Probabilistic MRI brain anatomical atlases based on 1,000 Chinese subjects. PLoS One 2013;8:e50939 CrossRef Medline 